\title{
Artificial Intelligence and Virtual Environment for Microalgal Source for Production of Nutraceuticals
}

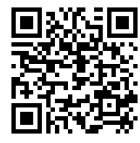

\author{
Archana Pattanaik ${ }^{1}$, Lala Behari Sukla*1, Debabrata Pradhan ${ }^{1}$ and Vinita Shukla ${ }^{2}$ \\ ${ }^{1}$ Biofuels and Bioprocessing Research Center, ITER, Siksha 'O' Anusandhan (Deemed to be University), Bhubaneswar, India \\ ${ }^{2}$ Aievol Consulting Limited, London
}

Received: 阱January 22, 2019; Published: 制January 30, 2019

*Corresponding author: Lala Behari Sukla, Biofuels and Bioprocessing Research Center, G-Block, Institute of Technical Education and Research, Siksha 'O' Anusandhan (Deemed to be University), Jagamara, Khandagiri, Bhubaneswar-751030, Odisha, India

\begin{abstract}
With the growing implementation with the AI and virtual environment there is a better scope for the production of highest quality of nutraceuticals. Microalgae, capable of performing photosynthesis, are important for life on earth which provides a well-balanced mixture of nutrients to the organisms higher in the food web. Microalgae are considered as a potential source of protein, carbohydrate, fatty acids, vitamins, amino acids, carotenoids, phycobilliproteins, astaxanthin, lutein and antioxidants which provide health benefits such as controlling blood pressure, strengthening immune system, reduction of coronary heart diseases, serves as anticancer agent, and antioxidant etc. Nutritional value of microalgae varies from species to species depending upon the culture conditions. Thus the biochemical composition of microalgae determines their nutritional quality and utilization as food. Several reports suggested the commercial use of microalgal strains such as Spirulina, Chlorella, Klamath, Dunaliella, Haematococcus and Aphanizomenon, as nutraceuticals and nutritional supplements. The advantage of using microalgae is its high productivity on arable and non-arable land; therefore, they don't compete with agricultural crop production. Nutritional value of microalgae and its industrial commercialization today is still in its infancy and can be seen as a gateway to a multibillion dollar industry.
\end{abstract}

Therefore, there is a huge scope for future research on the identified microalgal species with respect to their nutritional value for different groups of animals as a food supplement and other commercial applications under varied environmental conditions. Intense effort is now on all over the world to devise suitable systems to make large scale( Raceway pond/Photo bioreactor) cultivation and harvesting of these microscopic aquatic organisms, having nutritional potentiality, in a cost-effective manner.

Keywords: Microalgae; Nutraceutical; Virtualisation; Virtual Environment; Robotic Virtual Machine

\section{Introduction}

The developing industrialization has affected the lifestyle of human beings to a great extent. Food habitats in the lifestyle are mostly affected. The frequent consumption of packaged and junk foods, fast eating food habitats, long scheduled work, sedentary lifestyle, etc. has led to increase in prevalence of various diseases like diabetes, obesity, various cancer and cardiovascular diseases [1]. Therefore, there is an increasing global concern on implementation of healthy foods in diet that will help to maintain a healthy lifestyle [2]. Thus, to reduce the possibility of health problem consumers are preferring to use foods rich in adequate nutrients (nutraceuticals). The increasing demand for nutraceuticals is propelling its expansion in the global market $[3,4]$. The term Nutraceutical is coined by Stephen L. DeFelice in 1989, from 'nutrition' and 'phar- maceutical' [5]. Nutraceuticals are described as biologically active compounds possessing disease preventing and health promoting properties.
Nutraceuticals have proven to provide therapeutic benefit with lesser side effects compared to other therapeutic agents [6,7].

Nutraceuticals contain several products such as vitamins, minerals, enzymes, antioxidants, probiotics, etc. Depending upon their functions nutraceuticals are grouped as dietary supplements, medicinal food, functional foods, etc. [8]. More than 470 nutraceuticals and functional foods are being available in market, proven to provide health benefits [9]. Sources of nutraceuticals include animals ( polysaccharides), plants (vitamins, phytochemicals), marine sources (chitosan, glucosamine), and microbes (poly amino acids, etc.) [10]. Microalgae are considered as most promising resource for various bioactive compounds [11]. Microalgal metabolites are gaining interest in global nutraceutical market [12]. Health benefits provided by algal nutraceuticals are immunity improvement, maintaining teeth and bone health, brain development, weight loss, cho- 
lesterol reduction, maintaining heart health and digestive system, controlling blood pressure, antioxidant property, etc. [13].

\section{Microalgae as Source of Nutraceuticals}

Microalgae are rapidly growing photoautotrophic organisms having the ability of synthesizing a variety of bioactive compounds. These are considered as a sustainable resource for nutraceutical due to their higher productivity and ability to grow in arable and non-arable land potentially avoiding competition with agricultural crops. Moreover, microalgae play a key role in reducing atmospheric CO2 by photosynthesis thus mitigating GHG (greenhouse gas) effect [1]. Bioactive compounds produced by microalgae involves vitamins (A, B1, B2, B6, B9, B12, C, D, E, and H), minerals (sodium, potassium, calcium, zinc, copper, etc.), proteins, omega 6, pigments/carotenoids, polysaccharides, PUFAs, phenolic compounds and sterols [14]. To meet the increasing demand of nutraceutical production more research is required on the commercial implementation of primary and secondary metabolites of microalgal origin. Only few of the identified microalgal species are studied to evaluate their nutraceutical content. And a very less number of these studied species are being commercially exploited. There is a need for more research in isolating, identifying, and optimizing the growth of locally available microalgae species. Major challenge involves the high cost of production and recovery of desired compounds. To overcome this problem more research is needed in construction of raceway ponds/photobioreactors using cost intensive and ecofriendly materials. Energy efficient and inexpensive harvesting and extraction technique should be developed [14,15] (Figure 1).

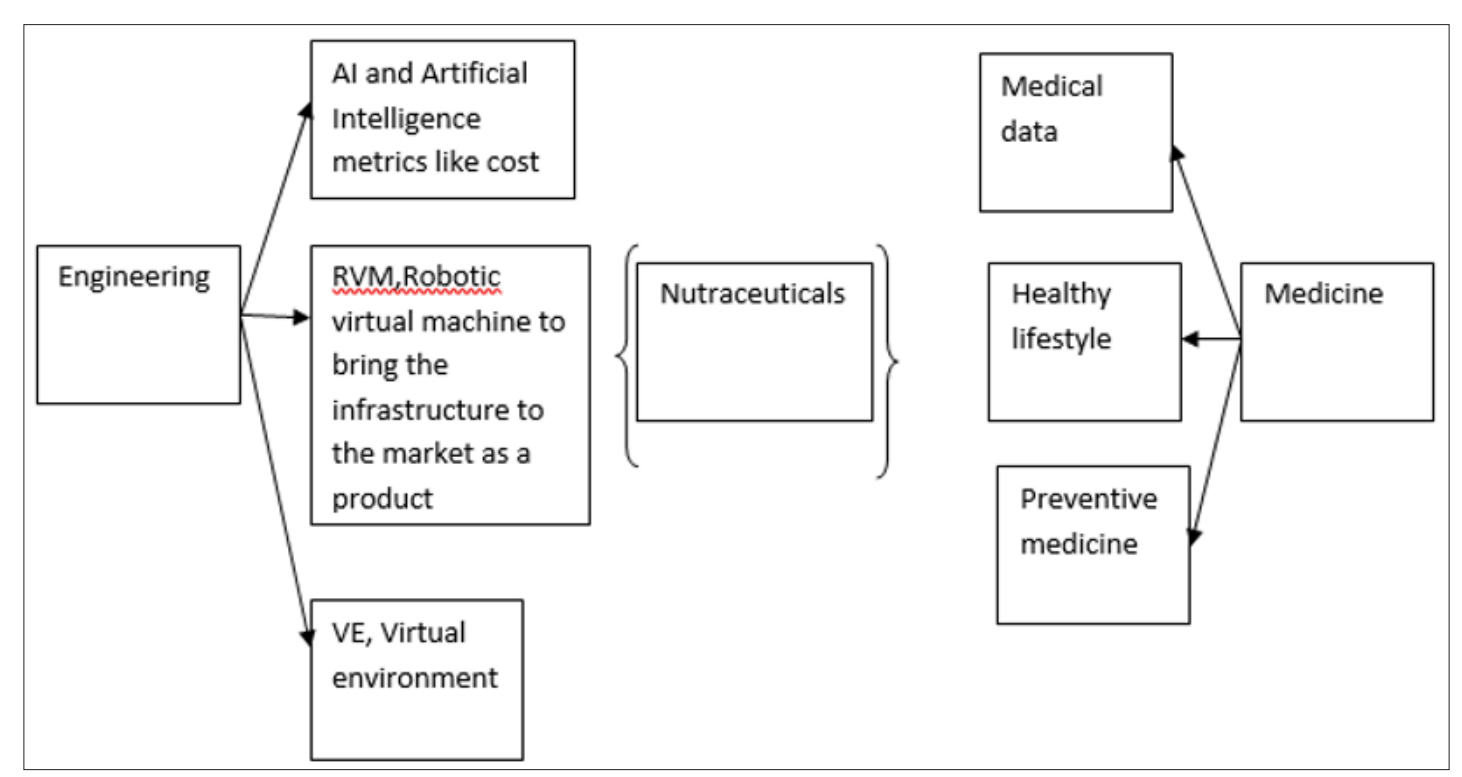

Figure 1: Diagram of relation of healthy lifestyle, preventive medicine and medical data when comes to picture AI, RVM and VE. Let us put the puzzle together for a complete picture as to role of $\mathrm{AI}$ in the role of nutracuetical procurement with better AI metrics.

\section{Nutraceutical Values of Different Microalgae}

Marcello Nicoletti reported three strains, i.e., Spirulina, Chlorella, and Klamath mostly used for marketed production of nutraceutical products. Dried spirulina used as food supplements consists of $60 \%$ of protein, which is superior to the protein derived from plants [1619]. West M. Bishop and Heidi M. Zubeck in their review explained the nutraceutical utilization and health benefits of Aphanizomenon, Chlorella, Spirulina Dunaliella, and Haematococcus [20]. Chlorella consists of protein (55-67\%), dietary fiber (9-18\%), and chlorophyll (1-4\%), including several vitamins and minerals [21]. Chlorella is known as large producer of lutein, which possess anti-cataract properties and used for treatment of macular degeneration [22]. Besides this Chlorella also possess antitumor [23,24], antioxidant [25], anti-inflammatory [26], and antimicrobial properties [27]. It also decreases cholesterol and blood pressure levels, improves immunity and fastens wound healing [28]. Dunaliella is documented to produce various carotenoids including alpha-carotene, beta- carotene, lycopene, and lutein [29]. Beta-carotene is an antioxidant which prevents several cancers (cervix, lungs, pancreas, stomach, rectum, colon, ovary, prostate and breast) [30].

Haematococcus is the highest producer of astanxanthin, comprising $1.5-3 \%$ of its dry weight [31]. Astaxanthin have 10 times stronger antioxidant property than beta-carotene and it is 1000 times more efficient when compared to vitamin E [32,33]. Besides its antioxidant property it protects human from UV radiation and oxidation of PUFAs, pigmentation, enhances immunity and vision, etc. [34-37]. It is used for the treatment of Parkinson's disease, Alzheimer's disease, and various central nervous system damages $[38,39]$. Any negative consequences upon the use of Haematococcus algal extract containing astaxanthin have not been reported [20]. Aphanizomenon contains chlorophyll i.e. 1-2\% of the dry weight (which increase bile secretion and stimulates liver functioning) [40], C-Phycocyanin, having anti-inflammatory and antioxidant properties. It also possess high hypo cholesterolemic activity 
(reduction of triglyceride and blood cholesterol levels) [41,42]. Aphanizomenon is reported to increase the number of natural killer cells which leads to the programmed cell death in cancerous and virus-infected cells [43].

The BCC Research shows that the global nutraceutical market grew to $\$ 46.7$ billion in 2002, at an AAGR (Annual Average Growth Rate) of nearly 7\%. In 2007 nutraceutical sale is projected to reach $\$ 74.7$ billion at an AAGR of 9.9\% [44]. The global nutraceuticals market is estimated at about $\$ 151$ billion in 2011 and it is estimated to reach nearly $\$ 207$ billion by 2016 at Compound Annual Growth Rate (CAGR) of 6.5\% between 2011 and 2016 [1,45]. Jose L Garcla et al. reported Spirulina to be the largest product of microalgal origin in the nutraceutical market with a production rate of 12,000 ton per year. About $70 \%$ of spirullina biomass is reported to be produced in India, Taiwan and China. Chlorella production is estimated to be 5000 tons per year. The market volume of other microalgae is followed by D. salina (3000 tons), A. flosaquae (1500 tons), $H$. pluvialis (700 tons), C. cohnii and Shizochytrium [46].

\section{Concept of Ai, Virtualization and Virtual Environment in The Field of Medical Data and Preventive Medicine For Better Lifestyle}

Time immemorial has iterative methods been success. The outcome being medicine and engineering research that have come a long way together with the evolution of Artificial intelligence, RVM [47] robotic virtual machine and virtual environment. Robotic virtual machine is a software and a software that will help find better medical data This software will replace the hardware and even physical robots as no pieces to lose [48] is the epitomy of any qualification. Virtual environment is for robotic virtual machine is the playground to play with different metrics like scalability. The success of virtualization lies in better results of the metrics like scalability, reliability and cost. After the success of virtualization of RVM, microalgae solution is inevitable with the stream of engineering and computers in it. Here replacement of the word computer with RVM and engineering stream along with the playground concept is called virtual environment. The success of microalgae is not independent of microalgae solution. The author of three publications on the newly coined word RVM robotic virtual machine and virtual environment would like to emphasize on the how critical its role in is reinventing the preventive medicine and medical data related to diagnosis and patient outcome result that this study is inclusive with the rest of health lifestyle and healthcare questions in this publication.

The mention of AI (artificial intelligence) is to importance lay down of relationship between the preventive medicine or techniques of treatment and the outcome result of patients. AI has helped analysis of study of the same. Study of concepts like AI, RVM and VE make this paper ever so enthralling that the readers would want for more. So here I go, Healthy lifestyle with its qualification suggest their capability in qualifying for VE.

\section{Conclusion}

With the increase in human population the demand for healthy and nutritive food products also increases. Microalgae have proven to be a great source of nutritive food that can meet the growing nutraceuticals demand. Microalgae are being utilized in pharmaceuticals, cosmetics, colorant, food, feed, aquaculture, nutraceuticals, etc. Researchers are aiming to search for microalgal strains with enhanced potential to accumulate high valued product. More research should be dedicated towards the development of cost intensive techniques of cultivation, harvesting and extraction. How is it related to its work? Thus, with the concept of the virtual environment the future of nutraceuticals is secure as the metrics are working well. AI has played an important role in analysis of medical data thereby encouraging healthy lifestyle. Thus, it is complementary and not supplementary as the jobs of human doctors would not be taken. The RVM intends to complement the work of human doctors who have been working in the field of preventive medicine and nutraceuticals. Also, it is the purpose of complementing their research and not banishes them.

\section{Acknowledgement}

The authors are grateful to Prof. (Dr.) Manojranjan Nayak, President, Siksha ' $O$ ' Anusandhan (Deemed To Be University), for providing infrastructure and encouragement throughout.

\section{References}

1. S Subudhi (2017) Bioprospecting for Algal Based Nutraceuticals and High Value Added Compounds. Journal of Pharmacy \& Pharmaceutics.

2. RA Ranga, RD Vijaya, GA Ravishankar (2017) Secondary Metabolites from Algae for Nutraceutical Application. Novel Techniques in Nutrition and Food Science 1(1).

3. J Zhao (2007) Nutraceuticals, nutritional therapy, phytonutrients, and phytotherapy for improvement of human health: A perspective on plant biotechnology application. Recent Pat Biotechnol 1(1): 75-97.

4. M Pandey, RK Verma, SA Saraf (2002) Nutraceuticals: new era of medicine and health. Asian J Pharmaceutical and Clinical Res 3(1): 1115.

5. SL DeFelice (1995) The nutraceutical revolution: its impact on food industry R\&D. Trends Food Sci Technol 6:59-61.

6. NJ Nelson (1999) Purple carrots, margarine laced with wood pulp? Nutraceuticals move into the supermarket. J Natl Cancer Inst 91(9): 755-757.

7. M Whitman (2001) Understanding the perceived need for complementary and alternative nutraceuticals: lifestyle issues. Clin J Oncol Nurs 5(5): 190-194.

8. CK Kokate, AP Purohit, SB Gokhale (2002) Nutraceutical and Cosmaceutical. Pharmacognosy, (21st Ed.) pp. 542-549.

9. NAM Eskin, S Tamir (2006) Dictionary of Nutraceuticals and Functional Foods. CRC Press, Boca Raton, FL USA.

10. J Wang, S Guleria, M Koffas, Y Yan (2016) Microbial production of valueadded nutraceuticals. Current Opinion in Biotechnology 37: 97-104.

11. O Pulz, W Gross (2004) Valuable products from biotechnology of microalgae. Appl Microbiol Biotechnol 65(6): 635-648. 
12. L Moreno-Garcia, K Adjalle, S Barnabe, GSV Raghavan (2017) Microalgae biomass production for a bio refinery system: Recent advances and the way towards sustainability. Renewable and Sustainable Energy Reviews 76: 493-506.

13. V Venugopal (2009) Marine Products for Healthcare: Functional and Bioactive Nutraceutical Compounds from the Ocean. CRC Press, Boca Raton, FL.

14. F Alsenani, F Ahmed, PM Schenk (2015) Nutraceuticals from Microalgae. Nutraceuticals and Functional Foods in Human Health and Disease Prevention pp. 673-682.

15. MA Borowitzka (2013) High-value products from microalgae-their development and commercialization. Journal of Applied Phycology 25: 443-756.

16. M Nicoletti (2016) Microalgae Nutraceuticals. Foods 5: 54.

17. MAB Habib, M Parvin, TC Huntington, MR Hasan (2008) A Review on Culture, Production and Use of Spirulina as Food for Humans and Feeds for Domestic Animals and Fish. Food and Agriculture Organization of the United Nations: Rome, Italy.

18. Z Khan, P Bhadouria, PS Bisen (2005) Nutritional and therapeutic potential of Spirulina. Curr Pharm Biotechnol 6: 373-379.

19. A Heidarpour, SAD Fourouzandeh, S Eghbalsaied (2011) Effects of Spirulina platensis on performance, digestibility and serum biochemical parameters of Holstein calves. Afr J Agric Res 6: 5061-5065.

20. WM Bishop, HM Zubeck (2012) Evaluation of Microalgae for use as Nutraceuticals and Nutritional Supplements. Nutrition \& Food Sciences 2: 5 .

21. JY Shim, HS Shin, JG Han, HS Park, BL Lim, KW Chung, AS Om (2008) Protective effects of Chlorella vulgaris on liver toxicity in cadmiumadministered rats. J Med Food 11: 479-485.

22. S Shibata, Y Natori, T Nishihara, K Tomisaka, K Matsumoto, et al. (2003) Antioxidant and anti-cataract effects of Chlorella on rats with streptozotocininduced diabetes. J Nutr Sci Vitaminol (Tokyo) 49: 334339.

23. F Konishi, K Tanaka, K Himeno, K Taniguchi, K Nomoto (1985) Antitumor effect induced by a hot water extract of Chlorella vulgaris (CE): resistance to Meth-A tumor growth mediated by CE-induced polymorphonuclear leukocytes. Cancer Immunol Immunother 19: 73-78.

24. K Tanaka, Y Tomita, M Tsuruta, F Konishi, M Okuda, et al. (1990) Oral administration of Chlorella vulgaris augments concomitant antitumor immunity. Immunopharmacol Immunotoxicol 12: 277-291.

25. MS Miranda, S Sato, J Mancini-Filho (2001) Antioxidant activity of the microalga Chlorella vulgaris cultered on special conditions. Boll Chim Farm 140: 165-168.

26. S Guzmán, A Gato, M Lamela, M Freire-Garabal, JM Calleja (2003) Antiinflammatory and immunomodulatory activities of polysaccharide from Chlorella stigmatophora and Phaeodactylum tricornutum. Phytother Res 17: 665-670.

27. T Hasegawa, K Tanaka, K Ueno, S Ueno, M Okuda, Y Yoshikai, K Nomoto (1989) Augmentation of the resistance against Escherichia coli by oral administration of a hot water extract of Chlorella vulgaris in rats. Int J Immunopharmacol 11: 971-976.

28. D Bewicke, BA Potter (1984) Chlorella: the emerald food. Ronin Publishing Berkley CA, USA.
29. KNC Murthy, A Vanitha, J Rajesha, MM Swamy, PR Sowmya, et al. (2005) In vivo antioxidant activity of carotenoids from Dunaliella salina-a green microalga. Life Sci 76: 1381-1390.

30. G Poppel, RA Goldbohm (1995) Epidemiologic evidence for betacarotene and cancer prevention. Am J Clin Nutr 62: 1393S-1402S.

31. RT Lorenz, GR Cysewski (2000) Commercial potential for Haematococcus microalgae as a natural source of astaxanthin. Trends Biotechnol 18: 160-167.

32. W Miki (1991) Biological functions and activities of animal carotenoids. Pure Appl Chem 63: 141-146.

33. YM Naguib (2000) Antioxidant activities of astaxanthin and related carotenoids. J Agric Food Chem 48: 1150-1154.

34. H Jyonouchi, S Sun, Y Tomita, MD Gross (1995) Astaxanthin, a carotenoid without vitamin A activity, augments antibody responses in cultures including T-helper cell clones and suboptimal doses of antigen. J Nutr 125: 2483-2492.

35. RG Fassett, JS Coombes (2012) Astaxanthin in cardiovascular health and disease. Molecules 17: 2030-2048.

36. JS Bertram (1999) Carotenoids and gene regulation. Nutr Rev 57: 182191.

37. M Bennedsen, X Wang, R Willén, T Wadström, LP Andersen (1999) Treatment of $H$. pylori infected mice with antioxidant astaxanthin reduces gastric inflammation, bacterial load and modulates cytokine release by splenocytes. Immunol Lett 70: 185-189.

38. MOM Tso, TT Lam (1996) Method of retarding and ameliorating central nervous system and eye damage. US Patent 5527533.

39. X Zhang, L Pan, X Wei, H Gao, J Liu (2007) Impact of astaxanthin-enriched algal powder of Haematococcus pluvialis on memory improvement in BALB/c mice. Environ Geochem Health 29: 483-489.

40. R Dashwood, D Guo (1995) Protective properties of chlorophylls against the covalent binding of heterocyclic amines to DNA in vitro and in vivo. Princess Takamatsu Symp 23: 181-189.

41. RI Kushak, C Drapeau, EM Van Cott, HH Winter (2000) Favorable effect of blue-green algae Aphanizomenon flos-aquae on rat plasma lipids. J Am Nutr Assoc 2: 59-65.

42. Miyamoto E, Tanioka Y, Nakao T, Barla F, Inui H, et al. (2006) Purification and characterization of a corrinoid-compound in an edible cyanobacterium Aphanizomenon flos-aquae as a nutritional supplementary food. J Agric Food Chem 54: 9604-9607.

43. R Manoukian, M Citton, P Huerta, B Rhode, C Drapeau (1998) Effects of the blue-green algae Aphanizomenon flos-aquae (L.) Ralphs on human Natural Killer cells. IBC Library Series, Thailand.

44. JL Garcia, M Vicente, B Galan (2017) Microalgae, old sustainable food and fashion nutraceuticals. Microbial Biotechnology.

45. V Shukla (2018) Recognition of virtual environment VE by robot virtual machine JETIR Research Journal 5(10): 115-116.

46. V Shukla (2018) Robotic virtual machine RVM. Robotic virtualisation future of RVM without having to lose the pieces like a physical robot, the new era and innovation JETIR Research Journal 5(11): 673-677. 
ISSN: 2574-1241

DOI: $10.26717 / B J S T R .2019 .13 .002459$

Lala Behari Sukla. Biomed J Sci \& Tech Res

(C) (P) This work is licensed under Creative

Submission Link: https://biomedres.us/submit-manuscript.php

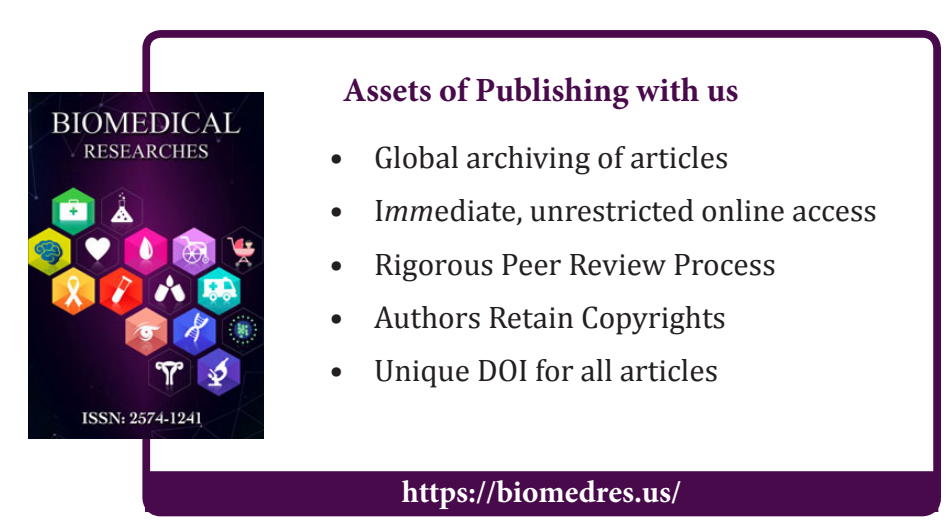

